\title{
Prevalência de sintomas persistentes em indivíduos infectados pelo novo coronavírus após 30 dias de diagnóstico
}

\section{Prevalence of persistent symptoms in individuals infected by the new coronavirus after 30 days of diagnosis}

\author{
Michelle Moreira Abujamra Fillis ${ }^{1}$ Larissa Laskoviski², Josiane Marques Felcar ${ }^{3}$ Celita \\ Salmaso Trelha ${ }^{4}$
}

1. ORCID: https://orcid.org/0000-0002-7457-3229. Fisioterapeuta. Doutorado. Secretaria de Saúde de Londrina.

E-mail: micmoreira@gmail.com

2. ORCID: https://orcid.org/0000-0002-7527-3471. Fisioterapeuta. Doutorado. Universidade Estadual de Londrina, Londrina, Paraná, Brasil. E-mail: larissal@uel.br

3. ORCID: https://orcid.org/0000-0003-3270-6940. Fisioterapeuta. Doutorado. Universidade Estadual de Londrina, Paraná, Brasil. E-mail: josianefelcar@uel.br

4. ORCID: https://orcid.org/0000-0001-5643-9002. Fisioterapeuta. Doutorado. Universidade Estadual de Londrina, Paraná, Brasil. E-mail: celita@uel.br

CONTATO: Autor correspondente: Michelle Moreira Abujamra Fillis | Endereço: Rua Eurico Hummig, 350 ap 103. Cep: 86050464. Londrina - Paraná, Brasil. Telefone: (43) 991615577 E-mail: micmoreira@gmail.com

\section{RESUMO}

Após infecção aguda pelo SARS-CoV 2 os pacientes podem apresentar sintomas persistentes e heterogêneos que afetam órgãos e sistemas. $O$ objetivo foi analisar a persistência de sintomas da COVID-19 após 30 dias do diagnóstico. Estudo transversal incluindo adultos maiores de 18 anos, ambos os sexos, com diagnóstico de COVID-19 em Londrina-PR. A coleta de dados foi por meio de questionário (WhatsApp) abordando aspectos demográficos, 
estado funcional e sintomas. Foram avaliados 1.021 indivíduos com mediana de idade de 34 [26-44] anos, boa escolaridade, 36,4\% tinham comorbidades, e poucos necessitaram de internação. Apresentaram limitação funcional $56,3 \%$ dos pacientes e, após um mês, $57,2 \%$ apresentavam fadiga e 34,8\% dispneia. Mulheres tiveram significativamente mais fadiga $61,7 \%$, dispneia $38,8 \%$ e maior limitação funcional $65,3 \%$. Homens tiveram mais internação e maior relato de sintomas. Concluiu-se que $73,5 \%$ dos participantes relataram permanência de ao menos um sintoma após 30 dias e os mais prevalentes foram fadiga, anosmia e cefaleia.

DESCRITORES: Infecção pelo Coronavírus. COVID-19. Avaliação de sintomas.

\section{ABSTRACT}

After acute SARS-CoV 2 infection, patients may present persistent and heterogeneous symptoms affecting organs and systems. The objective was to analyze a persistence of symptoms of COVID-19 30 days after the diagnosis of the disease. Cross-sectional study including 1,021, over 18 years old, both sexes, diagnosed with COVID-19 in Londrina-PR. Data collection was carried out through a questionnaire (WhatsApp) addressing demographic aspects, functional status and symptoms. The patients had a median age of 34 [26-44] years, good education, $36.4 \%$ had comorbidities and few needed hospitalization. Men progressed more fatigue $61.7 \%$, dyspnea $38.8 \%$, greater functional limitation $65.3 \%$. Women had more hospitalization and more reports of symptoms. Functional limitations were found in $56.3 \%$ of patients. After one month, $57.2 \%$ had fatigue and $34.8 \%$ had dyspnea. It was concluded that $73.5 \%$ of the participants reported the presence of at least one symptom after 30 days and the most prevalent were fatigue, loss of smell and headache.

DESCRIPTORS: Coronavirus Infections. COVID-19. Symptom Assessment. 


\section{INTRODUÇÃO}

O novo tipo de coronavírus foi detectado em dezembro de 2019 na China, o SARS-CoV 2, propagou-se rapidamente e tornou-se uma pandemia em pouco mais de dois meses. No início de 2020, a Organização Mundial da Saúde (OMS) adotou oficialmente o nome COVID-19 para se referir a doença causada pelo novo coronavírus e que se configura, principalmente, por infecções respiratórias que variam de condições leves e autolimitadas a distúrbios graves, como pneumonia e comprometimento fisiológico sistêmico ${ }^{1}$.

O primeiro caso confirmado de COVID-19 no Brasil foi em 26 de fevereiro de 2020, em São Paulo, um homem de 61 anos, com histórico de viagem para a Itália, e em apenas 11 dias a soma dos casos confirmados atingiu 25 pessoas ${ }^{2}$. Em 17 de março do mesmo ano, foi registrado o primeiro óbito pela doença no país ${ }^{3}$.

Dados de novembro de 2021 apontam que a epidemia de COVID-19 afetou mais de 200 países (áreas ou territórios) com um total de 252.902 .685 casos confirmados e 5.094.826 mortes no mundo ${ }^{4}$. No Brasil, até novembro de 2021, os casos de COVID-19 ultrapassavam 21 milhões e contabilizavam mais de 611 mil mortes $^{5}$. Os dados da Secretaria de Saúde do Estado do Paraná (SESA) ${ }^{6}$ apontavam para 1.563 .131 casos positivos e 40.482 óbitos no estado do Paraná no mesmo período e, na cidade de Londrina, onde há uma população de quase 570 mil habitantes, foram mais de 87 mil os casos acumulados de pacientes contaminados, com 2.299 mortes no total ${ }^{7}$.

Em contrapartida, os dados da SESA ${ }^{6}$ de novembro de 2021, apontam para recuperação de 1.485 .953 casos no estado do Paraná ${ }^{6}$ e, em Londrina ${ }^{7}$, foram aproximadamente 85.161 mil casos recuperados.

A infecção por SARS-CoV 2 pode ser assintomática ou pode causar um espectro amplo de sintomas, desde queixas leves de infecção de vias aéreas superiores até manifestações que ameaçam a vida ${ }^{8}$. Segundo a OMS, os sintomas da COVID-19 são febre, tosse seca, cansaço, perda do olfato ou paladar, cefaleia, distúrbios gastrointestinais, entre outros já relatados ${ }^{9}$.

O termo "long Covid", traduzido como Covid prolongado", é usado para descrever pessoas que se recuperam da infecção pelo vírus, mas ainda relatam efeitos duradouros ou apresentam sintomas por mais tempo do que o esperado. Nalbandian e colaboradores (2021) em uma recente revisão, consideraram a COVID 
pós-aguda como a persistência de sintomas ou o desenvolvimento de sequelas após quatro semanas de início dos sintomas da doença. Os autores dividiram a COVID pósaguda em duas categorias: COVID-19 subaguda ou persistente com sintomas presentes entre 4-12 semanas e COVID-19 Crônica ou Pós-COVID-19 quando os sintomas se estendem além de 12 semanas ${ }^{10}$. Um estudo de coorte prospectivo apresentou relatos de prejuízos moderados na qualidade de vida de pacientes que foram hospitalizados até três meses após o início dos sintomas ${ }^{11}$.

Diante do exposto, justifica-se a investigação da persistência de sintomas pósCOVID-19 de forma a possibilitar o acompanhamento e tratamento adequados destes pacientes com sintomas prolongados. O objetivo desta pesquisa foi investigar a persistência de sintomas de COVID-19 após 30 dias a partir da data de diagnóstico, em adultos da cidade de Londrina, na região sul do Brasil.

\section{MÉTODO}

Trata-se de um estudo transversal parte de um projeto maior intitulado "Avaliação clínica funcional e qualidade de vida de pacientes após 1, 2, 6 e 12 meses do diagnóstico de infecção por SARS-CoV 2 do município de Londrina". Foi desenvolvido e conduzido em parceria entre a Universidade Estadual de Londrina e a Secretaria Municipal de Saúde de Londrina - Paraná.

A pesquisa teve autorização da Secretaria Municipal de Saúde e aprovação do Comitê de Ética em Pesquisa envolvendo Seres Humanos da Universidade Estadual de Londrina (UEL) CAAE 36782620.0.0000.5231, Parecer ํㅜ 4.235.042. Todos os participantes receberam informações sobre a pesquisa e concordaram com a pesquisa de acordo com o Termo de Consentimento Livre e Esclarecido.

Os participantes foram recrutados mediante pesquisa na plataforma oficial da SESA de notificações de casos suspeitos de COVID-19 ("Notifica-COVID"), disponibilizado pela Secretaria Municipal de Saúde do município de Londrina.

Foram incluídos no estudo todos os indivíduos residentes de Londrina, com diagnóstico positivo pelo teste molecular PCR-RT para SARS-CoV 2, com idade maior ou igual a 18 anos, que concordaram com a pesquisa. Foram excluídos da pesquisa os indivíduos com ausência de elucidação de agente etiológico, sem possibilidade de contato, pessoas privadas de liberdade, idosos em Instituições de Longa Permanência e pessoas com dificuldades no uso de tecnologia digital ou acesso à internet. 
O cálculo do tamanho da amostra foi feito pelo programa GPower 3.1 utilizando a escala FSS, tamanho de efeito de $10 \%$, significância de $5 \%$ e poder de $80 \%$. O número de participantes necessário para 30 dias após o diagnóstico foi de 620 pacientes. Considerando uma perda de $20 \%$ corresponde a uma amostra de 744 pessoas.

A coleta de dados foi realizada no período de 12 de outubro de 2020 a 12 de março de 2021. Neste período, 11.178 londrinenses foram diagnosticados com COVID-19 por meio do teste PCR- RT e, destes, 1.021 responderam ao formulário e compõem a amostra do presente estudo ${ }^{7}$.

Os participantes receberam o questionário (Google Forms), por meio de aplicativo de conversa (WhatsApp), 30 dias após o diagnóstico de infecção por SARSCoV 2 e consistiu em dados sociodemográficos (sexo, idade e escolaridade), hospitalização, estado funcional pós-COVID-19, fadiga, dispneia e demais sintomas. Os instrumentos eram autoaplicáveis e havia explicação sobre como responder em cada um deles. Caso houvesse dúvidas, os pesquisadores estavam à disposição para respondê-las pelo WhatsApp.

O estado funcional foi avaliado pela Escala de Estado Funcional Pós-COVID19 (PCFS), instrumento elaborado para monitorar a recuperação direta, avaliar sequelas funcionais e classificar a capacidade em desempenhar atividades diárias e laborativas após infecção. A escala é ordinal, contém seis níveis que variam de zero (sem sintomas) a cinco (morte), e compreende desfechos funcionais, com foco nas limitações de tarefas e atividades de vida diária, bem como as mudanças de estilo de vida $^{12}$.

Na PCFS o Grau 0 (zero) reflete a ausência de qualquer limitação funcional. Graus 1 e 2 correspondem a quais tarefas/atividades diárias poderiam ser executadas, e essas são definidas como quaisquer atividades que o paciente realiza mensalmente ou mais frequentemente, seja em casa ou no trabalho/estudo, importante ressaltar que atividades esportivas e sociais estão incluídas. Grau 3 refere-se a limitações funcionais moderadas, que obrigam os pacientes a modificarem estruturalmente as atividades diárias, refletindo na incapacidade em realizar certas atividades, necessitando, portanto, que elas sejam assumidas por outras pessoas e o grau 4 descreve aqueles pacientes com limitações funcionais graves que necessitam de assistência para as Atividades de Vida Diária (AVD) ${ }^{13}$. 
A escala foi traduzida para a língua Portuguesa Brasileira e está em processo de validação. Apresentou validade do construto em indivíduos adultos altamente sintomáticos com COVID-19 confirmado ou presumido três meses após o início dos sintomas $^{14}$.

Foi utilizada a Escala de Severidade de Fadiga (FSS) que se trata de um questionário com nove afirmações onde o paciente escolhe um número de um a sete que melhor descreva o grau de concordância com cada afirmação. O número um significa que discorda completamente, o número sete que concorda integralmente, sendo o número quatro indicativos de que o paciente não concorda nem discorda da afirmativa ${ }^{15}$. O número total de pontos poderá variar de nove a 63 , sendo estabelecido que valores iguais ou maiores do que 28 são indicativos da presença de fadiga ${ }^{16}$.

Para avaliar a dispneia utilizou-se a Escala de Borg Modificada, instrumento que permite avaliar a dispneia tanto em repouso como quanto à intensidade do exercício, em termos de determinações de índices subjetivos, conforme a percepção do indivíduo, proporcionando diretamente uma medida individualizada da percepção da dispneia. A escala foi modificada da original em 1982 passando a ser pontuada de 0 a 10 pontos, com expressões verbais na qual cada número corresponde a uma intensidade de falta de ar, desde "nenhuma" até "máxima". É reprodutível e válida na avaliação da dispneia ${ }^{17}$.

Após o preenchimento do formulário, os participantes receberam uma cartilha de orientações e exercícios baseada em material elaborado pela OMS que provê o suporte para reabilitação autogerenciada após SARS-CoV $2^{18}$. Nesta cartilha constam informações sobre: gerenciamento da falta de ar, exercícios físicos, orientações sobre problemas de voz, dificuldades para atividades de vida diária, alterações cognitivas, mudanças de humor e estresse e contatos de emergências.

Para a análise estatística foram utilizados os softwares SPSS versão 27 (IBM, NY, EUA) e Graphpad Prism 6 (Graphpad, CA, USA). O teste de Shapiro-Wilk foi utilizado para analisar a normalidade na distribuição das variáveis numéricas. Como as mesmas não atingiram seus pressupostos, foram descritas como mediana e intervalo interquartílico 25-75. As variáveis categóricas foram apresentadas por meio de frequência absoluta e relativa.

Para comparar as variáveis numéricas entre os grupos (gênero) foi utilizado o teste de Mann-Whitney. Para avaliar a associação entre as variáveis categóricas foi utilizado o teste do qui quadrado (com ou sem correção de Yates) ou teste exato de 
Fisher. Para a correlação entre as variáveis foi utilizado o coeficiente de correlação de Spearman ${ }^{19}$. As correlações foram consideradas fortes $(0,70 \leq r<0,90)$, moderadas $(0,50 \leq r<0,70)$ e fracas $(0,30 \leq r<0,50)$ A significância estatística adotada foi de $P<0,05$.

\section{RESULTADOS}

Foram analisados 1.021 pacientes que responderam ao questionário no período de 12 de outubro de 2020 a 12 de março de 2021. A maioria dos pacientes era jovem, com boa escolaridade, sem comorbidades e não precisou de internação hospitalar, sendo $65,4 \%$ do sexo feminino e $34,6 \%$ do masculino (Tabela 1). 0 paciente mais jovem tinha 18 anos e o mais idoso 85 anos. Quanto ao tempo de internação, o mínimo foi um dia e o máximo 23 dias.

Tabela 1. Caracterização da amostra

\begin{tabular}{lcccc}
\hline \multicolumn{1}{c}{ VARIÁVEL } & $\begin{array}{c}\text { TOTAL } \\
\mathrm{N}=1.021\end{array}$ & $\begin{array}{c}\text { FEMININO } \\
\mathrm{N}=668\end{array}$ & $\begin{array}{c}\text { MASCULINO } \\
\mathrm{N}=353\end{array}$ & $\boldsymbol{P}$ \\
\hline IDADE* & $34[26-44]$ & $33[26-42]$ & $37[27-47]$ & $0,001^{* *}$ \\
FAIXA ETÁRIA (N; \%) & & & & \\
Menos de 60 anos & $965(94,5)$ & $641(96,0)$ & $324(91,8)$ & $0,005^{* *}$ \\
Maior ou igual 60 anos & $56(5,5)$ & $27(4,0)$ & $29(8,2)$ & \\
ESCOLARIDADE (N; \%) & & & & \\
Pós-graduação completa & $242(23,7)$ & $159(23,8)$ & $83(23,5)$ & 0,147 \\
Ensino médio completo & $233(22,8)$ & $156(23,4)$ & $77(21,8)$ & \\
Superior completo & $213(20,9)$ & $130(19,5)$ & $83(23,5)$ & \\
Superior incompleto & $179(17,5)$ & $110(16,5)$ & $69(19,5)$ & \\
Pós-graduação incompleta & $46(4,5)$ & $33(4,9)$ & $13(3,7)$ & \\
Ensino médio incompleto & $46(4,5)$ & $32(4,8)$ & $14(4,0)$ & \\
Outros & $62(6,1)$ & $48(7,1)$ & $14(4,0)$ & \\
INTERNAÇÃo (N; \%) & $56(5,5)$ & $16(2,4)$ & $40(11,3)$ & $\leq 0,001^{* *}$ \\
Sim & $965(94,5)$ & $652(97,6)$ & $313(88,7)$ & \\
Não & $0[0-0]$ & $0[0-0]$ & $0[0-0]$ & $\leq 0,001^{* *}$ \\
DIAS DE INTERNAÇÃO* & & & & \\
COMORBIDADES (N; \%) & $372(36,4)$ & $257(38,5)$ & $115(32,6)$ & 0,063 \\
Sim & $649(63,6)$ & $411(61,5)$ & $238(67,4)$ & \\
Não &
\end{tabular}

Fonte: Elaborada pelos autores (2021); ${ }^{*} \mathrm{Md}\left[1^{\circ}\right.$ e $\left.3^{\circ} \mathrm{q}\right]$; ${ }^{* *} \mathrm{P} \leq 0,05$ 
Após 30 dias do diagnóstico da COVID-19, a maioria apresentava fadiga avaliada pela Escala FSS e tinham alteração da funcionalidade por meio da Escala PCFS, entretanto, somente 34,8\% referiram apresentar dispneia quando avaliados pela Escala de Borg (Tabela 2). Homens tiveram significativamente mais fadiga (escala FSS), dispneia (escala de Borg), maior limitação funcional, entretanto, as mulheres apresentaram mais internação e relato de sintomas.

Tabela 2. Dispneia, fadiga e funcionalidade após 30 dias do diagnóstico de COVID-19

\begin{tabular}{lcccc}
\hline \multicolumn{1}{c}{ VARIÁVEL } & TOTAL & FEMININO & MASCULINO & $\boldsymbol{P}$ \\
& $\mathrm{N}=1.021$ & $\mathrm{~N}=668$ & $\mathrm{~N}=353$ & \\
\hline DISPNEIA (N; \%) & & & & \\
Sim & $355(34,8)$ & $259(38,8)$ & $96(27,2)$ & $\leq 0,001^{* *}$ \\
Não & $666(65,2)$ & $409(61,2)$ & $257(72,8)$ & \\
ESCALA DE BORG* & $0[0-0,5]$ & $0[0-1]$ & $0[0-0,5]$ & $\leq 0,001^{* *}$ \\
FADIGA (N; \%) & & & & \\
Sim & $584(57,2)$ & $412(61,7)$ & $172(48,7)$ & $\leq 0,001^{* *}$ \\
Não & $437(42,8)$ & $256(38,3)$ & $181(51,3)$ & \\
ESCALA FSS & & & & \\
LIMITAÇÃo FUNCIONAL(N; \%) & & & & \\
Sim & $575(56,3)$ & $436(65,3)$ & $139(39,4)$ & $\leq 0,001^{* *}$ \\
Não & $441(43,2)$ & $230(34,4)$ & $211(59,8)$ & \\
ESCALA PCFS (N; \%) & & & & \\
Grau 0: nenhuma limitação & $441(43,2)$ & $230(34,4)$ & $211(59,8)$ & $\leq 0,001^{* *}$ \\
Grau 1: limitação muito leve & $206(20,2)$ & $146(21,9)$ & $60(17,0)$ & \\
Grau 2: limitação leve & $270(26,4)$ & $218(32,6)$ & $52(14,7)$ & \\
Grau 3: limitação moderada & $76(7,4)$ & $56(8,4)$ & $20(5,7)$ & \\
Grau 4: Limitação grave & $23(2,3)$ & $16(2,4)$ & $7(2,0)$ & \\
\hline
\end{tabular}

Fonte: Elaborada pelos autores (2021); ${ }^{*} \mathrm{Md}\left[1^{\circ}\right.$ e $\left.3^{\circ} \mathrm{q}\right]$; ${ }^{* *} \mathrm{P} \leq 0,05$

Em relação aos sintomas, da amostra total, 1.008 (98,7\%) apresentaram sintomas durante a vigência da doença e 680 (66,6\%) permaneceram com algum sintoma após 30 dias do diagnóstico (Figura 1). Os pacientes apresentaram uma mediana de 8 [5-11] sintomas durante a COVID-19 e de 1 [0-3] após 30 dias. 
Figura 1. Sintomas persistentes após 30 dias de diagnóstico de COVID-19

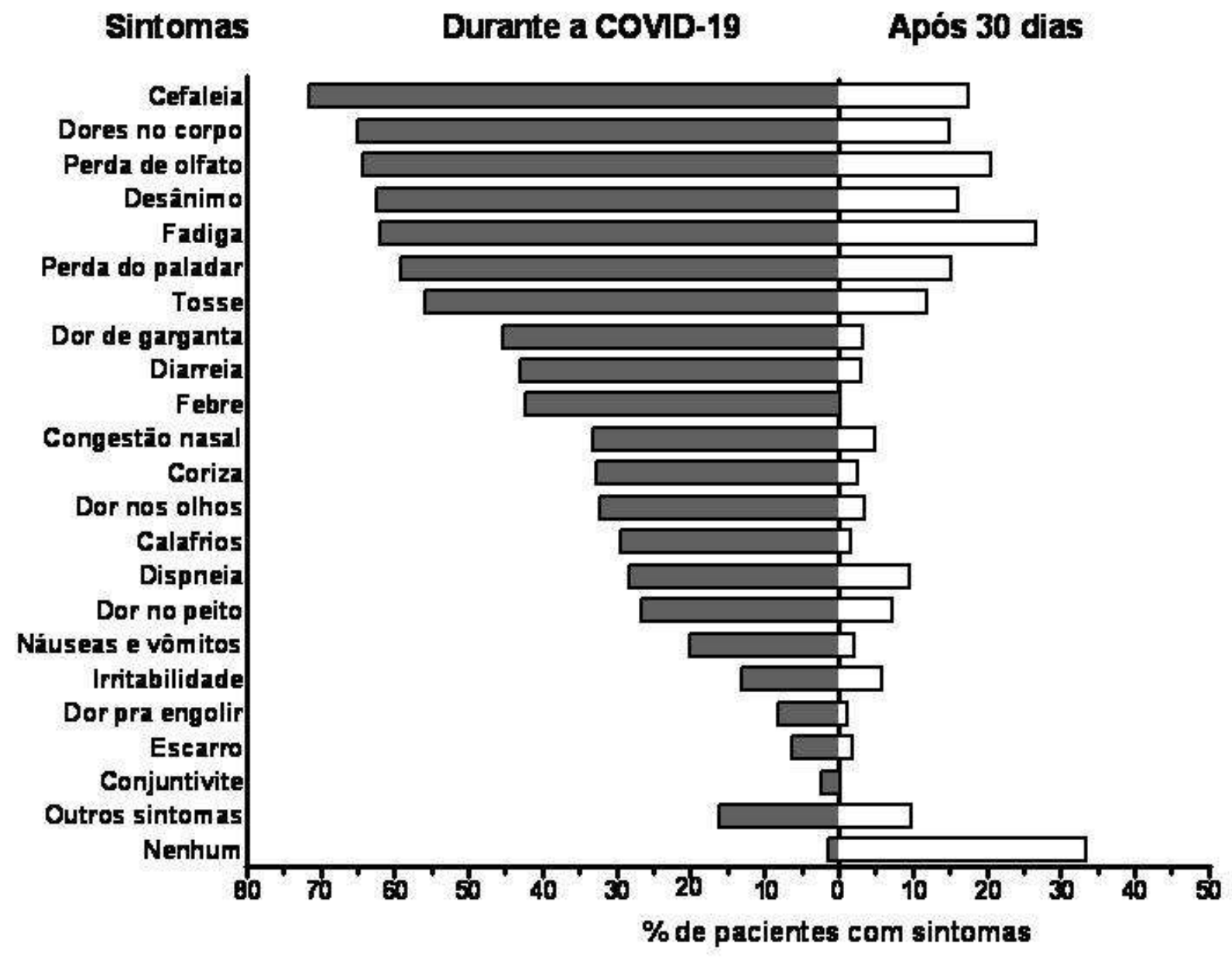

Fonte: Elaborada pelos autores (2021)

Os sintomas iniciais e após 30 dias separados por sexo podem ser visualizados na Tabela 3.

Tabela 3. Sintomas durante e após 30 dias da COVID-19 separados por sexo

\begin{tabular}{|c|c|c|c|c|c|c|c|c|c|c|}
\hline \multirow{3}{*}{ SINTOMAS } & \multicolumn{5}{|c|}{ Iniciais } & \multicolumn{5}{|c|}{ Após 30 dias } \\
\hline & \multicolumn{2}{|c|}{ FEMININO } & \multicolumn{2}{|c|}{ MASCULINO } & \multirow[t]{2}{*}{$P$} & \multicolumn{2}{|c|}{ FEMININO } & \multicolumn{2}{|c|}{ MASCULINO } & \multirow[t]{2}{*}{$P$} \\
\hline & $\mathbf{N}$ & $\%$ & $\mathbf{N}$ & $\%$ & & $\mathbf{N}$ & $\%$ & $\mathbf{N}$ & $\%$ & \\
\hline Cefaleia & 522 & 78,1 & 208 & 58,9 & $\leq 0,001^{*}$ & 145 & 21,7 & 34 & 9,6 & $\leq 0,001^{*}$ \\
\hline Desânimo & 477 & 71,4 & 160 & 45,3 & $\leq 0,001^{*}$ & 115 & 17,2 & 50 & 14,2 & 0,208 \\
\hline $\begin{array}{l}\text { Dores no } \\
\text { corpo }\end{array}$ & 458 & 68,6 & 206 & 58,4 & $0,001^{*}$ & 119 & 17,8 & 33 & 9,3 & $\leq 0,001^{*}$ \\
\hline Fadiga & 451 & 67,5 & 182 & 51,6 & $\leq 0,001^{*}$ & 189 & 28,3 & 85 & 24,1 & 0,148 \\
\hline Perda de olfato & 449 & 67,2 & 209 & 59,2 & $0,011^{*}$ & 159 & 23,8 & 52 & 14,7 & $0,001^{*}$ \\
\hline $\begin{array}{l}\text { Perda do } \\
\text { paladar }\end{array}$ & 413 & 61,8 & 190 & 53,8 & $0,013^{*}$ & 118 & 17,7 & 37 & 10,5 & $0,002^{*}$ \\
\hline Tosse & 377 & 56,4 & 193 & 54,7 & 0,590 & 80 & 12,0 & 41 & 11,6 & 0,865 \\
\hline
\end{tabular}




\begin{tabular}{|c|c|c|c|c|c|c|c|c|c|c|}
\hline $\begin{array}{l}\text { Dor de } \\
\text { garganta }\end{array}$ & 329 & 49,3 & 135 & 38,2 & $0,001^{*}$ & 28 & 4,2 & 6 & 1,7 & $0,035^{*}$ \\
\hline Diarreia & 323 & 48,4 & 117 & 33,1 & $\leq 0,001^{*}$ & 16 & 2,4 & 15 & 4,2 & 0,101 \\
\hline Febre & 246 & 36,8 & 186 & 52,7 & $\leq 0,001^{*}$ & 1 & 0,1 & 2 & 0,6 & 0,276 \\
\hline $\begin{array}{l}\text { Congestão } \\
\text { nasal }\end{array}$ & 256 & 38,3 & 84 & 23,8 & $\leq 0,001^{*}$ & 37 & 5,5 & 13 & 3,7 & 0,191 \\
\hline Coriza & 240 & 35,9 & 95 & 26,9 & $0,004^{*}$ & 20 & 3,0 & 6 & 1,7 & 0,212 \\
\hline Dor nos olhos & 233 & 34,9 & 96 & 27,2 & $0,012^{*}$ & 27 & 4,0 & 8 & 2,3 & 0,138 \\
\hline Calafrios & 219 & 32,8 & 81 & 22,9 & $0,001^{*}$ & 15 & 2,2 & 2 & 0,6 & $0,046^{*}$ \\
\hline Dispneia & 213 & 31,9 & 76 & 21,5 & $\leq 0,001^{*}$ & 70 & 10,5 & 29 & 8,2 & 0,245 \\
\hline Dor no peito & 209 & 31,3 & 63 & 17,8 & $\leq 0,001^{*}$ & 59 & 8,8 & 16 & 4,5 & 0,012 \\
\hline $\begin{array}{l}\text { Náusea/ } \\
\text { vômito }\end{array}$ & 163 & 24,4 & 41 & 11,6 & $\leq 0,001^{*}$ & 17 & 2,5 & 5 & 1,4 & 0,238 \\
\hline Irritabilidade & 96 & 14,4 & 37 & 10,5 & 0,079 & 38 & 5,7 & 21 & 5,9 & 0,865 \\
\hline $\begin{array}{l}\text { Dor para } \\
\text { engolir }\end{array}$ & 61 & 9,1 & 22 & 6,2 & 0,107 & 11 & 1,6 & 1 & 0,3 & 0,067 \\
\hline Escarro & 39 & 5,8 & 26 & 7,4 & 0,342 & 15 & 2,2 & 4 & 1,1 & 0,211 \\
\hline Conjuntivite & 17 & 2,5 & 6 & 1,7 & 0,387 & 0 & 0,0 & 1 & 0,3 & 0,346 \\
\hline $\begin{array}{l}\text { Outros } \\
\text { sintomas }\end{array}$ & 120 & 18,0 & 44 & 12,5 & $0,023^{*}$ & 79 & 11,8 & 22 & 6,2 & $0,004^{*}$ \\
\hline Nenhum & 5 & 0,7 & 8 & 2,3 & 0,730 & 177 & 26,5 & $\begin{array}{c}16 \\
4\end{array}$ & 46,5 & $\leq 0,001^{*}$ \\
\hline
\end{tabular}

Fonte: Elaborada pelos autores (2021); ${ }^{*} \mathrm{P} \leq 0,05$

\section{DISCUSSÃO}

No presente estudo verificou-se uma alta incidência de sintomas persistentes em pacientes com COVID-19 após 30 dias do diagnóstico, sem limitação funcional ou limitação leve. Os sintomas mais prevalentes durante a doença foram: cefaleia, dores no corpo e perda de olfato; e após trinta dias foram: fadiga, perda de olfato e cefaleia. Após um mês $57,2 \%$ apresentavam fadiga pela escala FSS e 34,8\% apresentavam dispneia pela Escala de Borg. A população era jovem (mediana de 34 anos), maioria do sexo feminino $(65,4 \%)$, a minoria tinha comorbidades $(36,4 \%)$ e poucos precisaram de internação $(5,5 \%)$.

O estudo apresenta uma população relativamente jovem, o que dá suporte ao fato de apresentarem epidemiologicamente menor propensão a doenças crônicas degenerativas e necessidade de hospitalização. Estudos apontam que algumas comorbidades são fatores de risco tanto para aumento da mortalidade por Covid-19, como para a síndrome pós-Covid (PASC). Nesse sentido, os sintomas persistentes 
pós-COVID-19 apresentados têm menor risco de serem secundários a essas doenças crônicas ${ }^{20}$.

O predomínio do sexo feminino também foi constatado em outro estudo no Brasil ${ }^{21}$, enquanto em Wuhan na China, $56 \%$ dos casos pertenciam ao sexo masculino ${ }^{22}$. Ressalta-se que as estimativas populacionais para 2020 mostram discreto predomínio do sexo feminino na população brasileira e também no município de Londrina 7 . Com relação à faixa etária houve predomínio da faixa de 30 a 39 anos. Esses achados são semelhantes ao encontrado em estudo realizado em Wenzhou, China, que apresentou $58,9 \%$ dos casos confirmados em indivíduos residentes com essa faixa etária ${ }^{23}$.

A maioria dos participantes deste estudo não necessitou de hospitalização, mas apresentaram sintomas prolongados após 30 dias do diagnóstico. $O$ exato mecanismo desses sintomas pós-COVID-19 ainda é obscuro. No entanto, pesquisadores descrevem possíveis mecanismos como a baixa resposta de anticorpos à infecção por SARS-CoV 2, resposta inflamatória prolongada a esta infecção, descondicionamento e reinfecção por SARS-CoV $2^{24}$.

Ao longo da pandemia por COVID-19, estudos já relataram a diversidade clínica que os pacientes podem manifestar quando estão com a doença ativa. Os sintomas variam desde tosse, febre, dispneia a alterações musculoesqueléticas como dor, alterações gastrointestinais, fadiga, entre outros ${ }^{1,9,25}$. Um estudo demonstrou que, em geral, os pacientes internados com COVID-19 apresentam os seguintes sinais e sintomas: febre $(83 \%-99 \%)$, tosse $(59 \%-82 \%)$, fadiga $(44 \%-70 \%)$, perda de peso $(40 \%-84 \%)$, dispneia $(31 \%-40 \%)$, produção de secreção $(28 \%-33 \%)$ e mialgias $(11 \%-35 \%)^{25}$, diferente do que foi encontrado em nosso estudo.

Neste estudo verificou-se que $73,5 \%$ dos participantes relataram permanência de ao menos um sintoma após 30 dias, principalmente fadiga $274(26,8 \%)$, anosmia, hiposmia ou parosmia $211(20,7 \%)$, cefaleia 179 (17,5\%), desânimo 165 (16,2\%), perda do paladar $155(15,2 \%)$, dores no corpo - musculares e articulares $152(14,9 \%)$, tosse $121(11,9 \%)$ e dispneia $99(9,7 \%)$.

A fadiga constitui um dos sintomas mais comuns em indivíduos infectados por SARS-CoV $2^{1,8,26}$. Um estudo sobre as características clínicas das pessoas infectadas demonstrou que este sintoma apareceu em até $69,6 \%$ dos $\operatorname{casos}^{23}$. Além disso, as 
pesquisas têm evidenciado que o SARS-CoV 2 tem o potencial de desencadear uma síndrome de fadiga pós-viral ${ }^{11,26}$.

No presente estudo foi possível perceber persistência da fadiga 30 dias após COVID-19, sendo que $57,2 \%$ da amostra apresentou pontuação maior que 28 no questionário FSS com mediana de 33 [16 - 49] pontos. Esses achados corroboram com a pesquisa em que os autores afirmaram que a fadiga não foi associada à gravidade inicial da doença ${ }^{24} \mathrm{e}$ ainda com estudo que explorou o nível de fadiga entre os médicos que trabalham após diagnóstico de COVID-19, com média de 36,7 $\pm 5,3$ na escala FSS ${ }^{26}$.

À medida que a pandemia de COVID-19 se espalhava pelo mundo, a anosmia e a disgeusia foram rapidamente reconhecidas como dois dos principais sintomas de apresentação e cerca de $25 \%$ dos pacientes relataram casos da persistência destes sintomas $^{27}$. No presente estudo, 64,4\% apresentaram anosmia na fase aguda e 20,7\% permaneceram com essa manifestação e 59,1\% apresentaram ageusia e 15,2\% persistiram com este sintoma 30 dias após o diagnóstico. A probabilidade de retorno do olfato está relacionada à gravidade da intensidade da perda na apresentação, mas parece que a perda do olfato e do paladar parece persistir em aproximadamente $10 \%$ dos pacientes afetados após seis meses ${ }^{27}$.

Dentre os achados neurológicos, a cefaleia lidera a lista de sintomas. Essa dor foi relatada em 11 a 34\% dos pacientes com diagnóstico de COVID-19 hospitalizados. Os possíveis mecanismos fisiopatológicos da cefaleia incluem a ativação das terminações nervosas trigeminais periféricas pelo SARS-CoV 2 diretamente ou através da vasculopatia e/ou aumento de citocinas pró-inflamatórias circulantes e hipóxia. Os autores referem também que as características mais marcantes da cefaleia foram de início súbito a gradual e má resposta aos analgésicos comuns, ou alta taxa de recidiva, que foi limitada à fase ativa do COVID-19. Além disso, cerca de $6 \%-10 \%$ dos pacientes sintomáticos com COVID 19 , relataram a cefaleia como um sintoma inicial da doença ${ }^{28}$.

$O$ vírus pode invadir diferentes tipos de tecidos do organismo e causar manifestações distintas de dor. O SARS-CoV 2 acomete vários sistemas e quando atinge o sistema respiratório os pacientes desenvolvem dor de garganta, febre, tosse e outros sintomas associados à pneumonia ${ }^{29}$

No sistema nervoso, além da cefaleia, pacientes podem desenvolver vertigem, zumbido e confusão; no sistema digestório pode ocasionar dor abdominal e diarreia, 
no sistema cardiovascular pode levar à dor no peito e lesão cardíaca. A taxa de incidência foi de 1,7-33,9\% para cefaleia, 0,7-47,1\% para dor de garganta, 1,5-61,0\% para mialgia / artralgia, 1,6-17,7\% para dor torácica e 1,9-14,5\% para dor abdominal. Diferentes dores refletem os danos de diferentes sistemas corporais ${ }^{29}$.

Além dos sintomas persistentes, o presente estudo verificou que $56,8 \%$ dos pacientes apresentaram limitação funcional verificada pela escala PCFS. A referida escala pode ser usada para medir o impacto dos sintomas sobre o estado funcional dos sujeitos após COVID-19, especialmente em categorias leves a severas ${ }^{13}$.

Os presentes achados descrevem que, provavelmente pela inclusão de uma amostra de indivíduos jovens, sem múltiplas comorbidades e com diagnóstico de COVID-19 "leve" (ou seja, não hospitalizados durante a infecção), a maioria deles (54\%) relatou grau de limitações de funcionalidade de muito leve a moderada durante o curso de COVID-19, 30 dias após o início dos sintomas. Pesquisadores demonstraram que $85 \%$ da sua amostra relatava função leve a moderada na escala PFCS, com perfil similar na caracterização da amostra com o presente estudo ${ }^{14}$.

Os autores destacam ainda a existência de uma 'Síndrome pós-COVID-19', que pode ser um alvo para decidir sobre o encaminhamento de indivíduos não hospitalizados para clínicas especializadas (ambulatoriais), facilitando a seleção de sujeitos que podem se beneficiar de programas de reabilitação, bem como medindo a eficácia de tais programas.

Durante o período de coleta de dados estava no início da implantação de serviços de reabilitação pós-COVID-19 no município. Portanto, este tipo de tratamento não influenciou nas respostas dos pacientes.

O estudo atual encontrou que as mulheres tiveram significativamente mais fadiga (escala FSS), dispneia (escala de Borg) e maior limitação funcional em relação aos homens. Em contrapartida, mais participantes do sexo masculino relataram maior internação e número de sintomas. Outro estudo com 444 participantes, no qual 56,8\% eram do sexo feminino, demonstrou que não houve diferença estatisticamente significante na limitação funcional pela PCFS entre homens e mulheres ${ }^{30}$.

O estudo tem como pontos fortes uma grande amostra, ser um dos poucos a demonstrar os sintomas e sua persistência em casos mais leves, bem como, destacase a alta escolaridade dos participantes que permitiu a compreensão dos questionários e respostas adequadas. Como limitação do estudo os sintomas, com exceção da fadiga e dispneia, foram autorrelatados pelos participantes, portanto os 
sintomas são subjetivos de acordo com a percepção de cada indivíduo. Também há o viés de responder o questionário somente quem tem acesso à internet e familiaridade com tecnologia digital.

\section{CONCLUSÃO}

A COVID-19 resultou em um quadro clínico de sintomas persistentes em 73,5\% dos pacientes acometidos 30 dias após o diagnóstico, mesmo nos indivíduos que não necessitaram de hospitalização durante a "primeira onda". Os sintomas prolongados mais comuns foram fadiga, anosmia, ageusia e cefaleia. A persistência de sintomas sobrecarrega ainda mais os sistemas de saúde, suas famílias e a sociedade em geral.

Os resultados deste estudo possibilitam conhecer as possíveis manifestações persistentes da COVID-19. Todos os sintomas devem ser considerados e analisados com precisão, inclusive os sintomas atípicos e leves, de maneira a direcionar melhor as estratégias de tratamento, recuperação e monitoramento contínuo desses pacientes.

Mais estudos são importantes para elucidação dessas manifestações persistentes a fim de identificar os mecanismos relacionados, a detecção precoce, a prevenção e o acompanhamento desses pacientes.

\section{REFERÊNCIAS}

1. Gulati A, Pomeranz C, Qamar Z, Thomas S, Frisch D, George G, et al. A Comprehensive Review of Manifestations of Novel Coronaviruses in the Context of Deadly COVID-19 Global Pandemic. Am J Med Sci. 2020;360(1):5-34. [acessado 12 jan 2021]. doi: https://doi.org/10.1016/j.amjms.2020.05.006

2. Macedo YM, Ornellas JL, Bomfim HF do. COVID - 19 NO BRASIL: o que se espera para população subalternizada? Encantar [Internet]. 2020 [citado 18 nov 2021];20:01-10. [acessado 12 Jan 2021]. Disponível em: https://revistas.uneb.br/index.php/encantar/article/view/8189.

3. Souza CDF, Paiva JPS, Leal TC, Silva LF, Santos LG. Evolução espaço temporal da letalidade por COVID-19 no Brasil, 2020 [carta ao editor]. J Bras Pneumol. 2020;46(4):e20200208. [acessado 07 Jan 2021]. doi: https://doi.org/10.36416/1806-3756/e20200208.

4. Coronavirus disease (COVID-19) situation reports [Internet]. [citado 18 nov 2021]. Disponível em: https://www.who.int/emergencies/diseases/novel-coronavirus2019/situation-reports. 
5. Brasil. Ministério da Saúde - Painel Coronavírus. [acessado 15 nov 2021]. Disponível em: https://covid.saude.gov.br.

6. Paraná. Secretaria de Saúde do Estado SESA. [acessado 15 nov 2021]. Disponível em: https://www.saude.pr.gov.br/Pagina/Coronavirus-COVID-19.

7. Autarquia Municipal de Saúde de Londrina. [acessado 15 nov 2021]. Disponível em: https://saude.londrina.pr.gov.br/index.php/dados-epidemiologicos/informeepidemiologico.html.

8. Wiersinga WJ, Rhodes A, Cheng AC, Peacock SJ, Prescott HC. Pathophysiology, Transmission, Diagnosis, and Treatment of Coronavirus Disease 2019 (COVID-19): A Review. JAMA. 2020;324(8):782-93. [acessado 28 jan 2021]. doi: https://doi.org/10.1001/jama.2020.12839.

9. World Health Organization (WHO). Origin of SARS-CoV-2, 26 March 2020. World Health Organization, 2020. [acessado 22 fev 2021]. Disponível em: https://apps.who.int/iris/bitstream/handle/10665/332197/WHO-2019-nCoV-FAQVirus origin-2020.1-eng.pdf.

10. Ladds E, Rushforth A, Wieringa S, Taylor S, Rayner C, Husain L, et al. Persistent symptoms after Covid-19: qualitative study of 114 "long Covid" patients and draft quality principles for services. BMC Health Serv Res. 2020;20(1):1-13. [acessado 22 fev 2021]. doi: https://doi.org/10.1186/s12913-020-06001-y.

11. Nalbandian A, Sehgal K, Gupta A, Madhavan MV, McGroder C, Stevens JS, et al. Post-acute COVID-19 syndrome. Nat Med. 2021;27(4):601-15. [acessado $02 \mathrm{fev}$ 2021]. doi: https://doi.org/10.1038/s41591-021-01283-z.

12. Wong AW, Shah AS, Johnston JC, Carsten C, Ryerson CJ. Patient-reported outcome measures after COVID-19: a prospective cohort study. Eur Respir J. 2020;56(5):2003276. [acessado 14 fev 2021]. doi: https://doi.org/10.1183/13993003.03276-2020

13. Klok FA, Boon GJAM, Barco S, Endres M, Geelhoed JJM, Knauss S, et al. The Post-COVID-19 Functional Status scale: a tool to measure functional status over time after COVID-19. Eur Respir J. 2020;56(1):2001494. [acessado em 24 Jan 2021]. doi: https://doi.org/10.1183/13993003.01494-2020.

14. Machado FVC, Meys R, Delbressine JM, Vaes AW, Goërtz YMJ, van Herck M, et al. Construct validity of the Post-COVID-19 Functional Status Scale in adult subjects with COVID-19. Health Qual Life Outcomes. 2021;19(1):1-10. [acessado $12 \mathrm{fev}$ 2021]. doi: https://doi.org/10.1186/s12955-021-01691-2.

15. Krupp LB, Larocca NG, Muir-Nash J, Steinberg AD. The fatigue severity scale: application to patients with multiples esclerosis and systemic lupus erythematosus. Arch Neurol. 1989,46(10):1121-3. [acessado 07 Fev 2021]. doi: https://doi.org/10.1001/archneur.1989.00520460115022.

16. Alvarenga Filho H, Carvalho SRS, Dias RM, Alvarenga RMP. Principais testes utilizados na avaliação de fadiga na esclerose múltipla: revisão sistemática. Rev 
Bras Neurol. 2010;46(2):37-43. [acessado $07 \mathrm{Fev}$ 2021]. Disponível em: http://files.bvs.br/upload/S/0101-8469/2010/v46n2/a0006.pdf.

17. Parshall MB, Schwartzstein RM, Adams L, Banzett RB, Manning HL, Bourbeau J, et al. An official American Thoracic Society statement: update on the mechanisms, assessment, and management of dyspnea. Am J Respir Crit Care Med. 2012;185(4):435-52. [acessado 07 fev 2021]. doi: https://doi.org/10.1164/rccm.201111-2042ST.

18. World Health Organization. Support for rehabilitation: self-management after COVID-19 related illness. No. WHO/EURO: 2021-855-40590-59892. World Health Organization. Regional Office for Europe, 2021. [acessado 15 nov 2021]. Disponível em: $\quad$ https://cdn.who.int/media/docs/default-source/ageing/support-forrehabilitation-self-management-after-covid-19-related-illness-engf5cec00b-350b4eb0-bc24-0704df509ae1.pdf?sfvrsn=203566f0 1\&download=true.

19. Mukaka MM. Statistics corner: A guide to appropriate use of correlation coefficient in medical research. Malawi Med J. 2012;24(3):69-71. [acessado 17 jan 2021]. Disponível em: https://pubmed.ncbi.nlm.nih.gov/23638278/

20.Pavli A, Theodoridou M, Maltezou HC. Post-COVID Syndrome: Incidence, Clinical Spectrum, and Challenges for Primary Healthcare Professionals. Arch Med Res. 2021;52(6):575-81. [acessado 16 nov 2021]. doi: https://doi.org/10.1016/j.arcmed.2021.03.010.

21. Rezer F, Faustino WR, Maia CS. Taxas de COVID-19 nas mesorregiões de Mato Grosso: casos confirmados e notificados. Rev Pre Infec e Saúde. [Internet] 2020;6:10317. [acessado 07 fev 2021]. Disponível em: https://revistas.ufpi.br/index.php/nupcis/article/download/10317/pdf

22. Li Q, Guan X, Wu P, Wang X, Zhou L, Tong Y, et al. Early Transmission Dynamics in Wuhan, China, of Novel Coronavirus-Infected Pneumonia. N Engl J Med. 2020;26382(13):1199-207. [acessado 07 fev 2021]. doi: https://doi.org/10.1056/NEJMoa2001316.

23. Han Y, Liu Y, Zhou L, Chen E, Liu P, Pan X, et al. Epidemiological Assessment of Imported Coronavirus Disease 2019 (COVID-19) Cases in the Most Affected City Outside of Hubei Province, Wenzhou, China. JAMA Netw Open. 2020;3(4):e206785. [acessado 07 fev 2021]. doi: https://doi.org/10.1001/jamanetworkopen.2020.6785.

24. Outhoff K. Sick and tired of COVID-19: long haulers and post viral (fatigue) syndromes [editorial]. South Afr Gen Pract J. 2020;1(4):132-3. [acessado $07 \mathrm{fev}$ 2021]. doi: https://doi.org/10.36303/SAGP.2020.1.4.0041.

25. Huang C, Wang Y, Li X, Ren L, Zhao J, Hu Y, et al. Clinical features of patients infected with 2019 novel coronavirus in Wuhan, China. Lancet. 2020;395(10223):497-506. [acessado 07 fev 2021]. doi: https://doi.org/10.1016/S0140-6736(20)30183-5.

26. Hasan ATMH, Islam MS, Khan N, Munna NH, Choton WR, Arefin MK, et al. Assessment of Post SARS CoV 2 Fatigue among Physicians Working in COVID 
Designated Hospitals in Dhaka, Bangladesh. medRxiv. 2021. [acessado $07 \mathrm{fev}$ 2021]. doi: https://doi.org/10.1101/2021.02.08.21251352

27. Davis HE, Assaf GS, McCorkell L, Wei H, Low RJ, Re'em Y, et al. Characterizing Long COVID in an International Cohort: 7 Months of Symptoms and Their Impact. medRxiv. 2020 [acessado 07 fev 2021]. doi: https://doi.org/10.1101/2020.12.24.20248802.

28. Bolay H, Gül A, Baykan B. COVID-19 is a Real Headache! Headache.. 2020;60(7):1415-21. [acessado 14 fev 2021]. doi: https://doi.org/10.1111/head.13856

29. Weng LM, Su X, Wang XQ. Pain Symptoms in Patients with Coronavirus Disease (COVID-19): A Literature Review. J Pain Res. 2021;14:147-59. [acessado $14 \mathrm{fev}$ 2021]. doi:https://doi.org/10.2147/JPR.S269206.

30. Mohamed-Hussein A, Galal I, Saad M, Zayan HE, Abdelsayed M, Moustafa M, et al. Post-COVID-19 Functional Status: Relation to age, smoking, hospitalization and comorbidities. medRxiv. 2020. [acessado $14 \mathrm{fev}$ 2021]. doi: https://doi.org/10.1101/2020.08.26.20182618.

RECEBIDO: $24 / 05 / 2021$

ACEITO: 16/12/2021 